\title{
UQ RoboRoos: Achieving Power and Agility in a Small Size Robot
}

\author{
Gordon Wyeth, David Ball, David Cusack, Adrian Ratnapala \\ School of Computer Science and Electrical Engineering \\ University of Queensland, Australia \\ wyeth@csee.uq.edu.au
}

\begin{abstract}
The UQ RoboRoos have been developed to participate in the RoboCup robot soccer small size league over several years. RoboCup 2001 saw a focus on the mechanical design of the RoboRoos, with the introduction of an omni-directional drive system and a high power kicker. The change in mechanical design had implications for the rest of the system particularly navigation and multi-robot planning. In addition, the overhead vision system was upgraded to improve reliability and robustness.
\end{abstract}

\section{Overview}

The RoboRoos are one of the longest serving teams in the RoboCup Small Size league. The robots competed at RoboCup '98 where they were runner-up and in RoboCup '99 where they were eliminated in the quarter finals, despite a 56-1 goal difference during the round robin stage. In RoboCup 2000, the team was eliminated in the semi-finals after a 36 - 2 goal difference in the round robin stage. In RoboCup 2001 , the team was eliminated by a "golden goal" in extra time during the quarter finals. Again, the team performed well in the round robin stage of the contest, achieving a 31 - 2 goal difference.

RoboCup 2001 saw a new look mechanical design for the RoboRoos. New robot bases were built featuring an omni-directional drive system. This base supported a new style of kicking device for the Small Size league: a cross-bow kicker capable of propelling the ball at $5 \mathrm{~m} / \mathrm{s}$. These new features and their impact on the RoboRoos system is the focus of this team description. The drive system and kicker design are described after an overview of the system architecture. Following the description of the mechanical improvements, a brief description of improvements to the vision system is given. The conclusion focuses on the future of the RoboRoos.

\subsection{System Architecture}

Figure 1 shows the architecture of the RoboRoos system, which has remained constant since its inception. The vision system was extensively revised in 1999 with 
excellent results [5], and was revised again in 2001. The new system uses the YUV color space, adapted from many of the principles and techniques used in the RGB color space described in [5]. This change and a new marking scheme on the robots has lead to improvements in accuracy, robustness and reliability that will be described in a later section.

The team planning system, MAPS, has been the focus of ongoing development 2 . 3. 4, and represents one of the most significant research results of the RoboRoos development. MAPS forms cooperative strategies by observing team agents at the current point in time and choosing appropriate actions to increase the likelihood of cooperation in the near future. These actions are transmitted to the robots, along with the current state of the field, over the RF communications link. The RF communications link has received significant attention to ensure reliability under adverse conditions. The robots use the information from the communications link to set the current goal for navigation, and to build representations of obstacle maps for path planning. The navigation module for the robots has been another area of significant research effort [1, and provides the smooth controlled motion that is the signature feature of the RoboRoos.

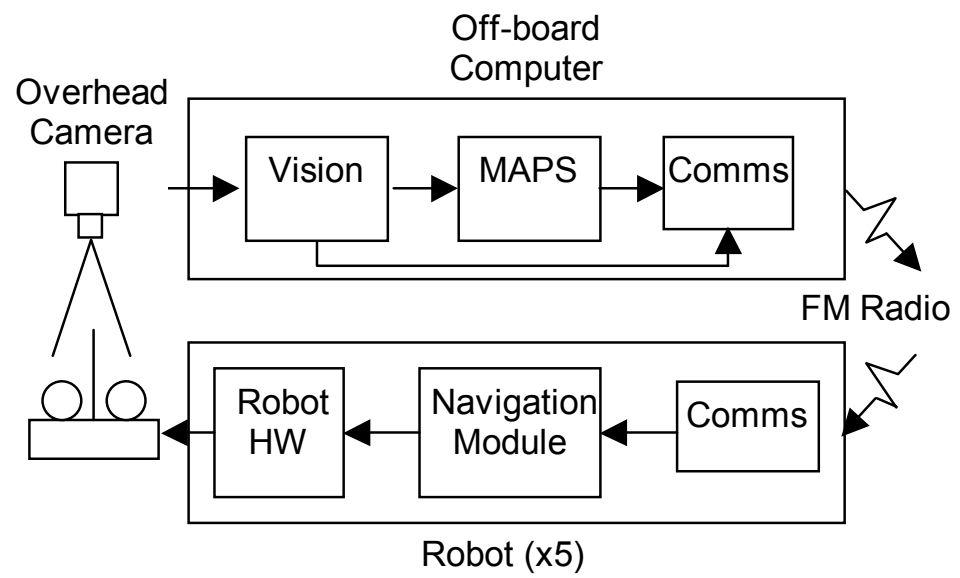

Figure 1. The RoboRoos system in overview.

\subsection{Omni-directional Drive System}

During RoboCup 2000, the team from Cornell illustrated an omni-directional drive system that allowed their robots to traverse in any direction while rotating at the same time. The maneuverability offered by their drive configuration showed clear advantages for soccer playing robots. During 2001, the RoboRoos team used the same principles for the design of new drive systems. The result is shown in Figure 2. The system is capable of accelerations up to $2.5 \mathrm{~ms}^{-2}$ while traversing at $2.2 \mathrm{~ms}^{-1}$, which is comparable to the speeds used in practice by the original RoboRoos field robots. The 
overall height of drive system and the height of the centre of gravity are also comparable to the original RoboRoos, keeping the load of the robot evenly distributed over the wheels during periods of acceleration. This ensures that the wheels have limited slip, and path integration information from the built-in encoders can maintain an accurate estimate of robot position and orientation between camera updates.

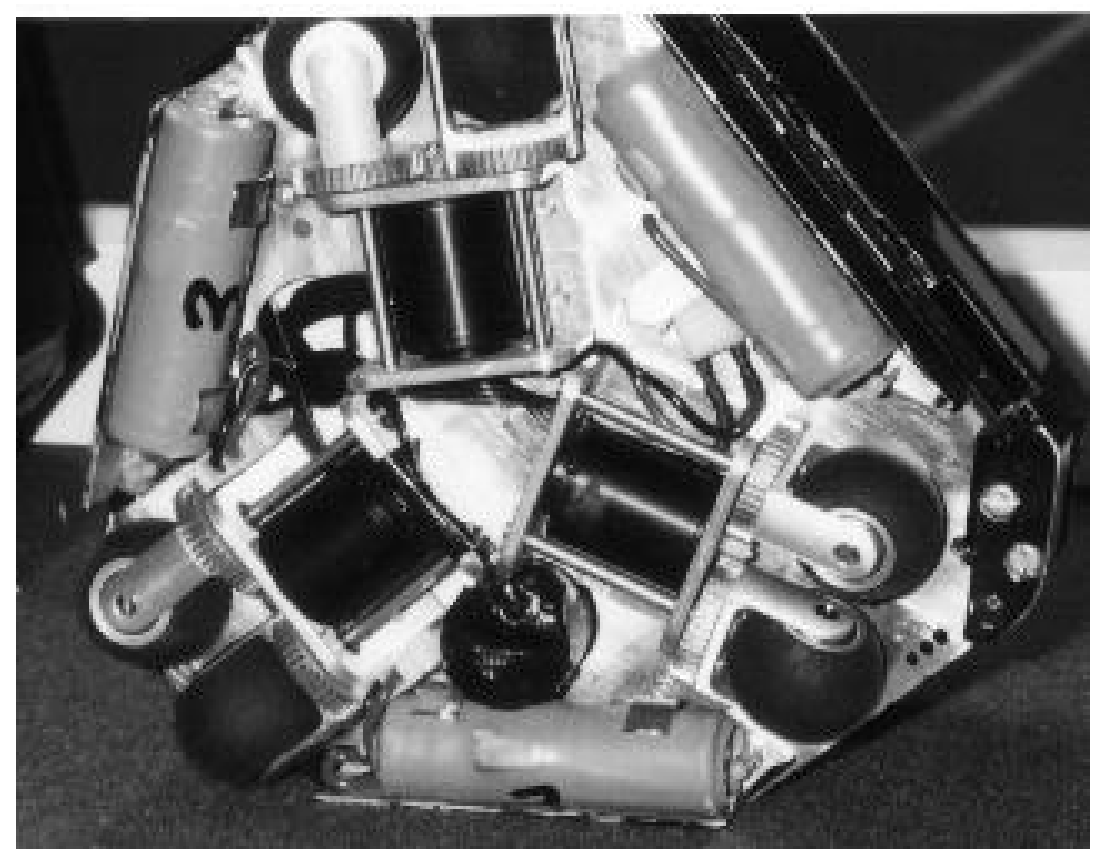

Figure 2. The omni-directional drive system of the RoboRoos 2001 robots. The custom made spherical wheels spin freely about one axis and are driven in the other. Each wheel set is made as a pair so that as one wheel breaks contact with the ground the other makes contact.

\subsection{Crossbow Kicker}

From analysis of games in previous tournaments, it was clear that existing kicker technology played a pivotal role in the success of the best teams. Our analysis indicated that a kicker capable of kicking the ball at $5 \mathrm{~ms}^{-1}$ was required to score goals against the best defenses in the competition. Designs using existing technologies (such as rotating blades, solenoids and servo drives) are not capable of transferring sufficient energy without a significant cost in weight and volume. The solution adopted by the RoboRoos is to have a crossbow mechanism, where the crossbow string is a cotton coated elastic cord. The cord pulls against the kicking blade which is the element that transfers the cord's stored energy to the ball. Energy is stored in the cord by driving the kicking blade using a rack and pinion mechanism from a DC motor. The blade can be locked in a number of positions by the trigger mechanism, so 
that kick strength can be controlled. The current design enables kicks between 2 and 5 $\mathrm{ms}^{-1}$. During the course of the competition, the kicker developed some mechanical failures, which meant that it could not be used at its full strength in the quarter-final in which the RoboRoos were defeated.

\subsection{Vision}

The RoboRoos vision system underwent a number of changes in 2001. The prime change was the use of YUV data rather than the RGB data. This change improved the robustness of the vision system further, particularly in relation to the classification of color markers used by some teams for robot identification. The RoboRoos black and white marking scheme was changed to help determine robot orientation. Whereas the previous robot shape allowed orientation to be determined from the overhead image of the robot, the symmetry of the new RoboRoos meant that a long white marker had to be placed on the black top of the robot. This scheme improved determination of orientation from \pm 7 degrees to \pm 1 degree, leading to improved efficiency in navigation and accuracy in shooting.

\section{RoboRoos 2002}

The RoboRoos introduced a number of changes in the team in 2001, most of which had not been fully tested or explored before the competition. In 2002, the RoboRoos will continue to test and develop the new mechanisms and improve the software that controls them. In addition, the RoboRoos will also experiment with ball control devices such as dribbling bars and explore the possibilities of a possession oriented game.

\section{References}

1. Browning B., Wyeth G.F. and Tews A. (1999) A Navigation System for Robot Soccer. Proceedings of the Australian Conference on Robotics and Automation (ACRA '99), March 30 - April 1, Brisbane, pp. 96-101.

2. Tews A. and Wyeth G.F. (2001) MAPS: A System for Multi-Agent Coordination. Advanced Robotics, VSP / Robotics Society of Japan, accepted for publication.

3. Tews A. and Wyeth G.F. (2000) Thinking as One: Coordination of Multiple Mobile Robots by Shared Representations, Proceedings IROS 2000.

4. Tews A. and Wyeth G.F. (1999) Multi-Robot Coordination in the Robot Soccer Environment. Proceedings of the Australian Conference on Robotics and Automation (ACRA '99), March 30 - April 1, Brisbane, pp. 90-95.

5. Wyeth G.F. and Brown B. (2000) Robust Adaptive Vision for Robot Soccer, Mechatronics and Machine Vision, Research Studies Press, pp. 41 - 48. 